\title{
Evolución del estreñimiento en pacientes que utilizan laxantes estimulantes de manera crónica cuando se les introduce un suplemento de fibra
}

\author{
Carlos Benavent Núñez ${ }^{1}$, Juan Carlos López García², Carlos González³ , Tamara Perulero4 ${ }^{4}$ Beatriz Izquierdo , \\ Ruth Delgado 6 \\ 1. Doctor en Farmacia por la Universidad Complutense de Madrid. Farmacéutico adjunto en Oficina de farmacia Carmen Núñez Martín. 2. Especialista \\ en Aparato Digestivo. Gastroenterología y Endoscopia Digestiva. 3. Farmacéutico adjunto en Oficina de farmacia Ángeles Nebot Rico. 4. Farmacéutica \\ adjunta en Oficina de farmacia Susana Bereciartúa. 5. Farmacéutica titular de Oficina de farmacia Verdaguer. 6. Asesora Médica, Departamento Médico \\ Laboratorios Casen Recordati.
}

\section{PALABRAS CLAVE}

Estreñimiento crónico, laxantes estimulantes, suplementos de fibra

\section{ABREVIATURAS}

AEMPS: Agencia Española de Medicamentos y Productos Sanitarios

\section{RESUMEN}

Objetivo: determinar la evolución del estreñimiento en pacientes que utilizan de forma crónica laxantes estimulantes cuándo se les introduce fibra.

Diseño: estudio epidemiológico de cohortes, multicéntrico y prospectivo de 10 semanas de seguimiento. Emplazamiento: el estudio se realizó en 16 farmacias comunitarias. Participantes: 106 pacientes con estreñimiento crónico y automedicados con laxantes estimulantes, a los cuales se les introduce fibra y se observa qué ocurre al retirar paulatinamente el laxante. Mediciones principales: Frecuencia defecatoria y consistencia de las heces. Se contabilizaron los laxantes requeridos y los sintomas gastrointestinales.

Resultados:a nivel basal, el 94,2\% de los pacientes necesitaban laxantes, el número medio de las deposiciones/ semana fue de 4,2 (DE 2,4) y solo el 4,8\% de los pacientes presentaron consistencia normal de las heces (valor 4 de la Escala de Bristol). Desde la primera semana de tratamiento con la fibra, hubo una disminución significativa del uso de laxantes estimulantes $(\mathrm{P}<0,02)$. De los pacientes que terminaron, menos de la mitad $(\mathrm{el} 41 \%)$ requirieron alguna dosis de laxante estimulante durante la última semana y un 59\% de los pacientes consiguieron dejar de tomarlos. Un 98,4\% de los pacientes valoraron como "bastante/mucho/muchísimo" la facilidad de uso de la fibra.

Conclusiones: en pacientes con estreñimiento que usan de manera crónica laxantes estimulantes, la incorporación paulatina de un suplemento de fibra mantiene la frecuencia de defecación del paciente y la consistencia normal de las heces, disminuyendo la necesidad del uso de laxantes estimulantes, cuyo uso debería restringirse a casos puntuales y no crónicos.

\section{INTRODUCCIÓN}

El estreñimiento es un cuadro clínico multifactorial que se diagnóstica según los criterios Roma IV (1). Es una situación clínica muy prevalente que afecta a entre un $2 \%$ y un $28 \%$ de la población general (2-5). En España la prevalencia es aún más alta y se estima en un $29,5 \%(6)$.

Existen muchas causas del estreñimiento, siendo una de ellas el abuso de laxantes estimulantes. Estos se dividen en 3 grupos:

1. Derivados antraquinónicos: provienen de plantas cuyos principios activos son glucósidos. Dentro del grupo están la cáscara sagrada, el sen, áloe, frángula y ruibarbo. Está demostrado que su uso crónico puede producir Melanosis coli que desaparece después de varios meses sin utilizarlos.

2. Laxantes polifenólicos: están constituidos por picosulfato y bisacodilo (7).

3. Aceite de ricino: su acción estimulante proviene del aceite ricinoleico (8).

Financiación: ninguna.

Conflicto de intereses: Ruth Delgado es asesora médica en Laboratorios Casen Recordati. El resto de autores declaran no tener ningún conflicto de intereses.

Cite este artículo como: Benavent C, López JC, González C, Perulero T, Izquierdo B, Delgado R. Evolución del estreñimiento en pacientes que utilizan laxantes estimulantes de manera crónica cuando se les introduce un suplemento de fibra. Farmacéuticos Comunitarios. 2022 Jan 11; 14 (1): 49-56. doi:10.33620/FC.2173-9218.(2022/Nol14).001.07

Correspondencia: Carlos Benavent Núñez (valdefarma@gmail.com).

ISSN 1885-8619 @SEFAC (Sociedad Española de Farmacia Clínica, Familiar y Comunitaria). Todos los derechos reservados. 
Existe controversia acerca de los efectos nocivos que el uso continuado de estos agentes produce sobre el plexo mientérico (9). En cualquier caso, se desaconseja un uso frecuente o prolongado por el riesgo de causar desajustes hidroelectrolíticos y debido a que pueden provocar dependencia o habituación (10-14). Su uso debería reservarse para tratar episodios agudos o como medicación de rescate.

Cuando la dosis y/o el tiempo de administración de estos laxantes es superior al recomendado se produce una habituación del plexo nervioso intestinal al estímulo, ya que el tono muscular intestinal y la respuesta de los nervios se pierde, produciéndose una dilatación del intestino y un peristaltismo inefectivo. Esto desencadena una dependencia al laxante, al requerirse una dosis cada vez más alta para evitar un cuadro agudo de estreñimiento. Estos síntomas no duran más de 1 a 3 semanas, pero producen muchas molestias y empeoramiento de la calidad de vida, que hacen difícil dejar la medicación. Por tanto, el abuso o uso indebido de este tipo de laxantes es causa directa de estreñimiento crónico $(15,16)$.

El tratamiento del estreñimiento crónico se ha de hacer con laxantes tipo incrementadores del bolo intestinal, como la fibra, o laxantes osmóticos. Este estudio se diseñó para investigar si aquellos pacientes con uso crónico de laxantes estimulantes conseguian dejar la medicación ayudándose del uso concomitante de un suplemento de fibra (Casenfibra ${ }^{\circledR}$.

\section{MATERIAL Y MÉTODOS}

Se realizó un estudio abierto, prospectivo de cohortes, de 2 meses y medio de duración y multicéntrico, en 16 farmacias comunitarias de la Comunidad de Madrid y Barcelona. Un total de 104 pacientes fueron analizados (figura 1).

El estudio fue clasificado por la Agencia Española de Medicamentos y Productos Sanitarios (AEMPS) y aprobado por el Comité Ético del Hospital Gregorio Marañón, de Madrid.

El estudio fue coordinado por un farmacéutico y por un gastroenterólogo.

\section{Población de estudio}

Se recogieron de forma consecutiva los primeros 6 pacientes que acudieron a la farmacia por cualquier motivo y que, tras cumplir con todos los criterios de inclusión y con ninguno de exclusión, firmaron el consentimiento informado.

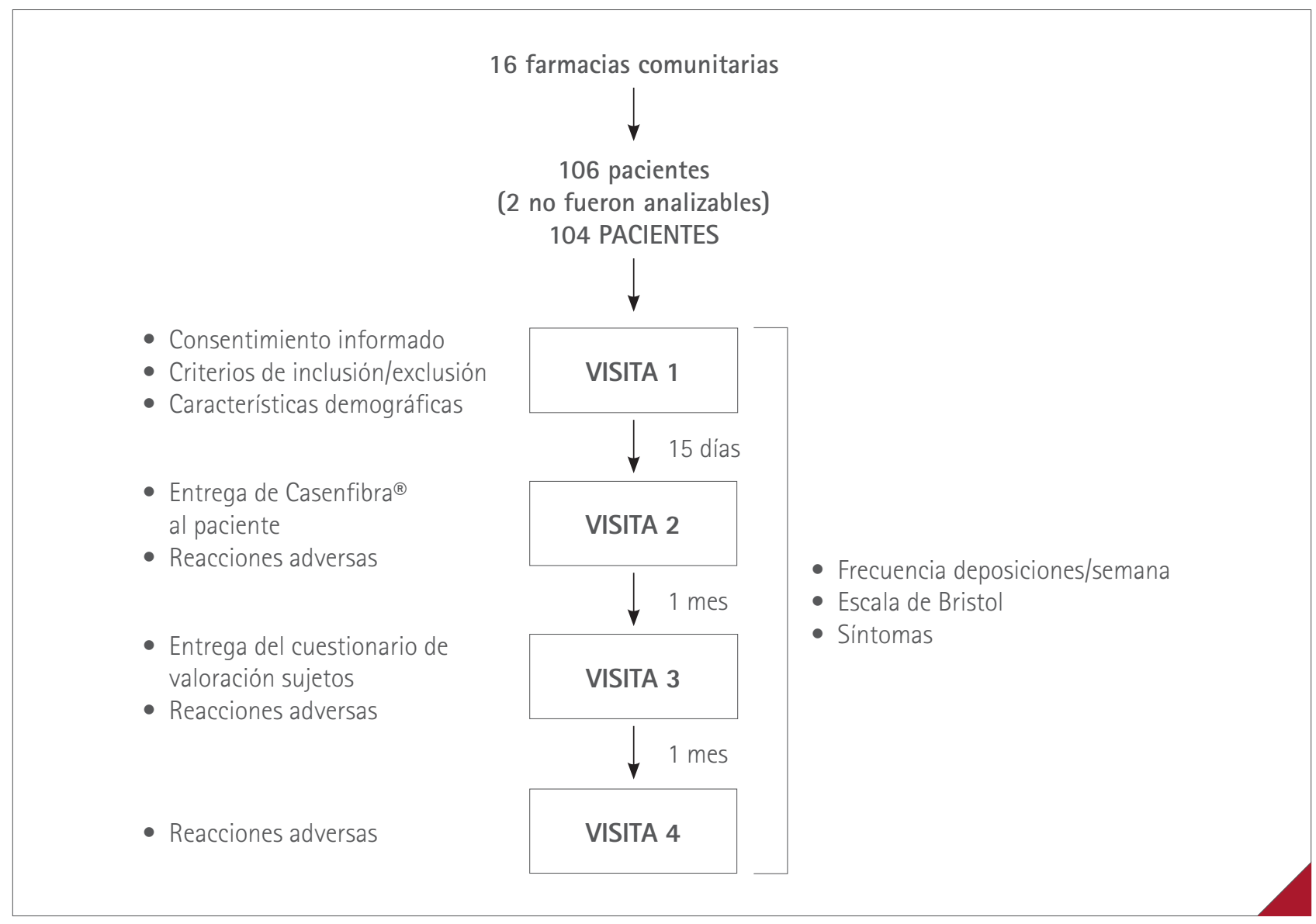

Figura 1 Esquema general del estudio 
Los criterios de inclusión fueron: pacientes de ambos sexos con edades entre los 18 y 75 años que estaban utilizando laxantes estimulantes (periodo mínimo de 30 días, aunque no necesariamente de manera consecutiva), y que eran habituales de la farmacia (al menos acudian 1 vez al mes durante los 4 meses anteriores al inicio del estudio de cada paciente).

A los farmacéuticos se les proporcionó un formulario diseñado por el gastroenterólogo coordinador para ayudarles a dar fiabilidad a las respuestas en relación a los criterios de exclusión: pacientes con sindrome de intestino irritable, enfermedad de Crohn, obstrucción intestinal, impactación fecal o dilatación del colon o con estreñimiento grave con importante enlentecimiento del tránsito colónico, que hubiesen tenido sangrados abundantes en los 4 últimos meses y pacientes con deterioro cognitivo.

A los sujetos incluidos se le entregó un diario y durante 15 días anotaron la consistencia de las deposiciones, sintomas intestinales y consumo de laxantes. A partir de entonces y durante las siguientes 8 semanas se les indicó que debían tomar un suplemento de fibra (Casenfibra ${ }^{\circledR}$ ) en una dosis máxima de 3 sobres/día. Se empezó con una dosis de 1 sobre/día incrementando después un sobre diario para mejor tolerancia. Durante este periodo, se siguió recogiendo en el cuestionario la misma información que a nivel basal. Igualmente, el paciente reflejaba en el diario si había tomado el suplemento de fibra. En caso negativo, señalaba el motivo. En caso afirmativo, el paciente indicaba el número de sobres que consumía diariamente.

Cuando los pacientes observaran una puntuación de 4 (o más) en la consistencia de las heces según la Escala de Bristol (figura 2), deberían disminuir paulatinamente la dosis de laxantes estimulantes hasta conseguir tomar solamente la fibra.

\section{Variables}

Se recogieron en una única visita los datos demográficos (fecha de nacimiento, género, peso y talla) y clínicos referentes a la etiología del estreñimiento del paciente y la utilización de laxantes estimulantes, así como los diferentes tratamientos farmacológicos utilizados durante el estudio.

Las variables principales fueron la variación del ritmo intestinal y la consistencia de las heces. Se cuantificó el número de deposiciones por semana, la consistencia de las heces mediante la escala de Bristol, el uso de laxantes (nombre comercial, frecuencia de uso y dosis) y síntomas gastrointestinales presentes: distensión abdominal, dolor y malestar, movimientos intestinales, si existía esfuerzo defecatorio prolongado o excesivo y si existía o no defecación insatisfactoria.

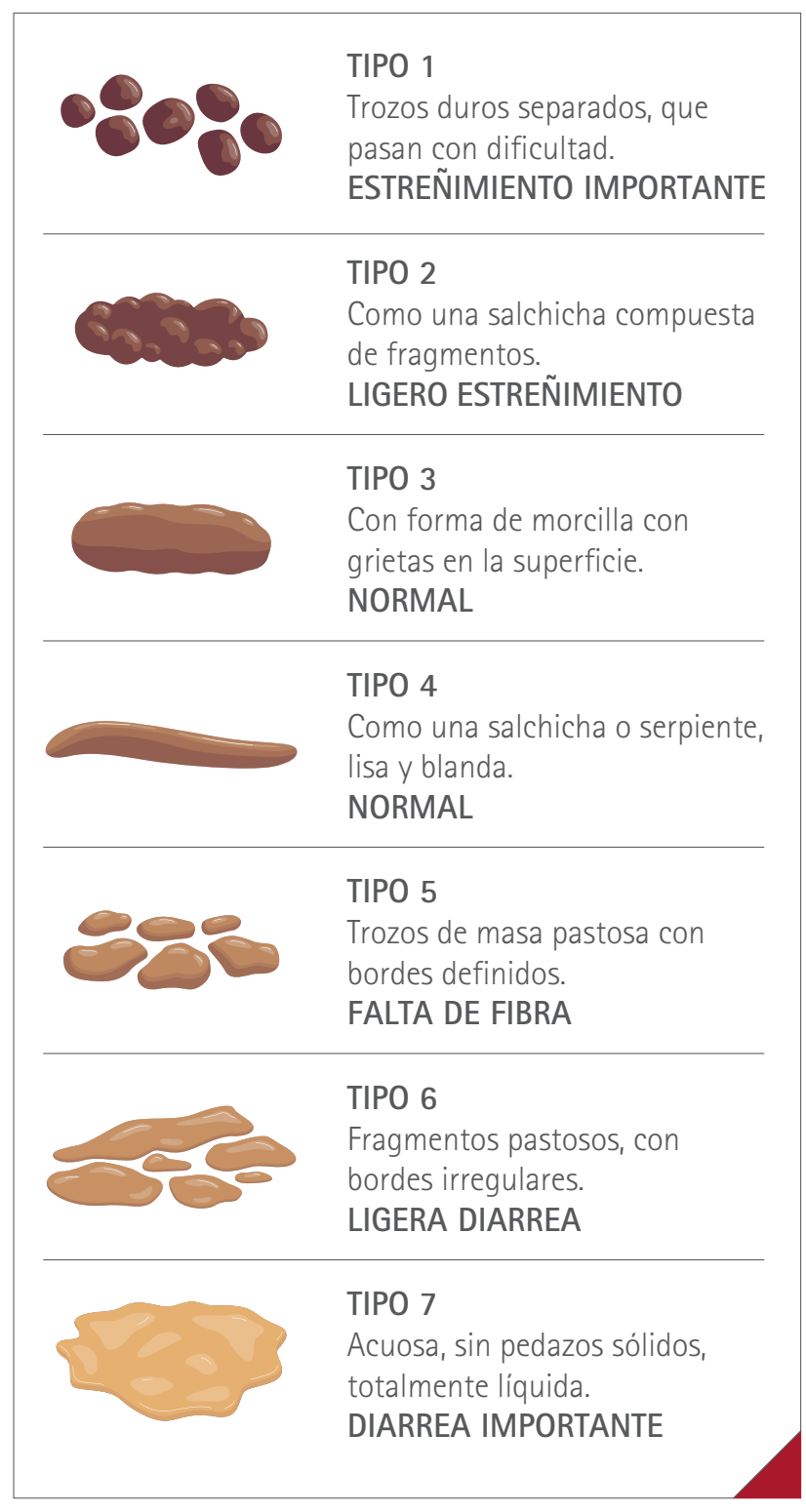

Figura 2 Escala de clasificación de tipos heces de Bristol

Se realizó un cuestionario a pacientes donde se les preguntó la eficacia que ellos observaban (eficacia subjetiva), la facilidad de administración de la fibra, aceptabilidad, valoración de la textura, sabor y grado de recomendación que harian del producto.

Los ítems del cuestionario de valoración global se agruparon en 3 categorías: Nada/casi nada; poco/algo; bastante/ mucho/muchísimo.

\section{Análisis estadístico}

La población de análisis incluyó todos los pacientes con datos, aunque no todos tenían datos del diario.

La estadística descriptiva de las variables se realizó incluyendo medidas de tendencia central y dispersión para las variables cuantitativas y frecuencias absolutas y relativas para las variables cualitativas. 
Referente a las variables del diario, se analizaron los resultados descriptivos por semana (desde la 0 hasta la 10). En ningún caso los datos perdidos se imputaron a partir de datos disponibles.

De los pacientes con datos completos, se calculó la significación estadística ( $p$-valor) de cada semana de seguimiento respecto a la semana 0 . Se aplicó la prueba de McNemar para las variables binarias, la prueba de la $t$ de Student para datos apareados en los datos cuantitativos, y para las variables ordinales la prueba de Mantel-Haenszel. No se realizaron ajustes para controlar el error de tipo I a pesar de presentar multiplicidad.

Se estudió el tipo de distribución de las variables y se evaluó su ajuste a la distribución de Gauss usando la prueba de Kolmogorov-Smirnov. Si los datos no cumplian el supuesto de normalidad, en los análisis se usaron métodos estadísticos no paramétricos.

Todas las pruebas estadísticas se realizaron con un nivel de significación del 5\%. (Figura 3)

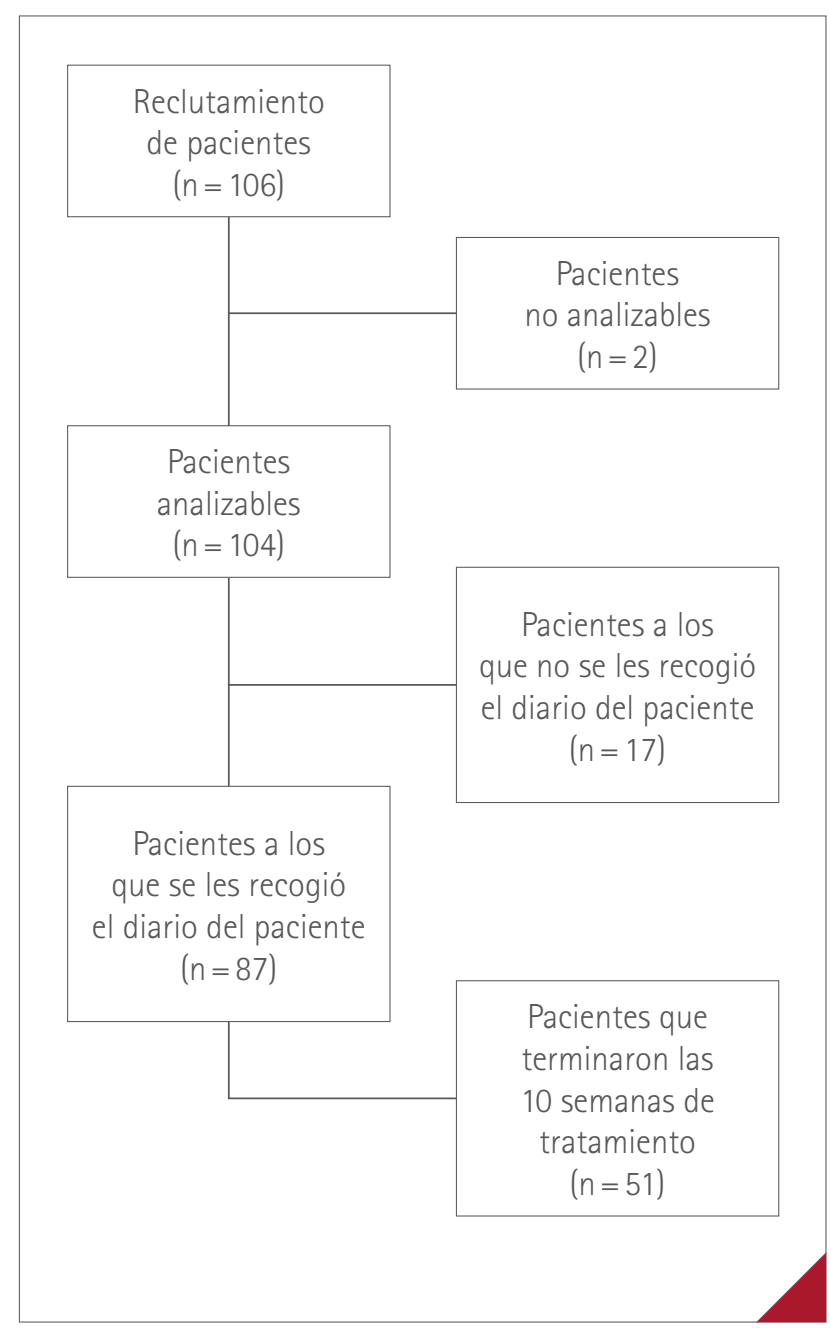

Figura 3 Flujograma de los pacientes del estudio

\section{RESULTADOS}

\section{Características de los pacientes}

Se reclutaron 106 pacientes de los que dos no fueron analizables. De los 104 finales, se recogió el diario en 87 (el $84 \%$ ). De ellos, 51 pacientes terminaron las 10 semanas de tratamiento.

La edad media fue de 52,6 $( \pm 13,4)$ años, siendo la mayoría mujeres $(90,4 \%)$ y con un IMC medio de 25,5 $( \pm 4,9) \mathrm{kg} / \mathrm{m}^{2}$.

Un 87,5\% afirmaron tomar suficientes alimentos ricos en fibra (verdura, fruta, legumbres y alimentos integrales).

A nivel basal, el tiempo de evolución del estreñimiento crónico fue igual o superior a 3 años en el $76 \%$ de los pacientes. El número medio de deposiciones semanal fue de $4,2( \pm D E 2,4)$ y solo el $4,8 \%$ de los pacientes presentaron consistencia normal de las heces. Los sintomas intestinales más frecuentes fueron: defecación insatisfactoria, esfuerzo defecatorio prolongado o excesivo, malestar y distensión abdominal, movimientos intestinales y dolor abdominal.

El $45,2 \%$ de los pacientes afirmaron sufrir estreñimiento a pesar de estar bajo tratamiento y el 94,2\% había utilizado al menos un laxante durante la última semana con una media de duración de 4,1 (DE 2,5) días. Los laxantes más utilizados fueron Dulcolaxo ${ }^{\circledR}$ (bisacodilo), Fave De Fuca ${ }^{\circledR}$ (Rhamnus frangula L, Rhamnus purshianus D.C y Fucus vesiculosus $L$., alga) y supositorios o microenemas de glicerina.

Se valoraron también los tratamientos concomitantes, ya que algunos de ellos pueden producir per se estreñimiento. Los más utilizados fueron el tramadol (6,9\%), omeprazol $(5,7 \%)$, atorvastatina, enalapril y simvastatina $(4,6 \%$ cada uno).

El $100 \%$ de los pacientes tomaron el suplemento de fibra durante todo el estudio, a excepción de la semana 4 (1 paciente), semana 6 ( 1 paciente), semana 8 (3 pacientes), 9 (2 pacientes) y 10 ( 2 pacientes). El motivo de la no administración de la misma fue la presentación de síntomas molestos como gases y acidez, olvido o encontrarse de viaje fuera de casa sin acceso a la fibra.

Los resultados muestran que el cambio respecto a nivel basal empezó a ser estadísticamente significativo $(p<0,05)$, tanto en la utilización como en el número de dias que se utilizaron laxantes estimulantes, a partir de la semana dos que coincide con el inicio de la toma de la fibra. En la tabla 1 se indica la utilización semanal de laxantes estimulantes. En la tabla 2 se muestran los datos solo de los 51 pacientes que completaron las 10 semanas de tratamiento y el número de días que estos 51 pacientes han utilizado laxantes estimulantes. 
Tabla 1 Utilización de laxantes estimulantes por semana

\begin{tabular}{|c|c|c|c|c|c|c|c|c|c|c|}
\hline Semana $\rightarrow$ & 1 & 2 & 3 & 4 & 5 & 6 & 7 & 8 & 9 & 10 \\
\hline \multicolumn{11}{|c|}{ Ha utilizado laxantes estimulantes } \\
\hline $\mathrm{N}$ & 78 & 62 & 73 & 66 & 62 & 58 & 62 & 57 & 58 & 51 \\
\hline No & $\begin{array}{c}10 \\
(12,8 \%)\end{array}$ & $\begin{array}{c}8 \\
(12,9 \%)\end{array}$ & $\begin{array}{c}23 \\
(31,5 \%)\end{array}$ & $\begin{array}{c}28 \\
(42,4 \%)\end{array}$ & $\begin{array}{c}31 \\
(50,0 \%)\end{array}$ & $\begin{array}{c}30 \\
(51,7 \%)\end{array}$ & $\begin{array}{c}35 \\
(56,5 \%)\end{array}$ & $\begin{array}{c}32 \\
(56,1 \%)\end{array}$ & $\begin{array}{c}33 \\
(56,9 \%)\end{array}$ & $\begin{array}{c}30 \\
(58,8 \%)\end{array}$ \\
\hline Sí & $\begin{array}{c}68 \\
(87,2 \%)\end{array}$ & $\begin{array}{c}54 \\
(87,1 \%)\end{array}$ & $\begin{array}{c}50 \\
(68,5 \%)\end{array}$ & $\begin{array}{c}38 \\
(57,6 \%)\end{array}$ & $\begin{array}{c}31 \\
(50,0 \%)\end{array}$ & $\begin{array}{c}28 \\
(48,3 \%)\end{array}$ & $\begin{array}{c}27 \\
(43,5 \%)\end{array}$ & $\begin{array}{c}25 \\
(43,9 \%)\end{array}$ & $\begin{array}{c}25 \\
(43,1 \%)\end{array}$ & $\begin{array}{c}21 \\
(41,2 \%)\end{array}$ \\
\hline \multicolumn{11}{|c|}{ Número de días que ha utilizado laxantes estimulantes } \\
\hline $\mathrm{N}$ & 78 & 62 & 73 & 66 & 62 & 58 & 62 & 57 & 58 & 51 \\
\hline $\begin{array}{l}\text { Media } \\
\text { (DE) }\end{array}$ & $\begin{array}{c}3,9 \\
(2,7)\end{array}$ & $\begin{array}{c}3,6 \\
(2,7)\end{array}$ & $\begin{array}{c}2,9 \\
(2,7)\end{array}$ & $\begin{array}{c}2,4 \\
(2,8)\end{array}$ & $\begin{array}{c}2,2 \\
(2,8)\end{array}$ & $\begin{array}{c}1,5 \\
(2,2)\end{array}$ & $\begin{array}{c}1,9 \\
(2,8)\end{array}$ & $\begin{array}{c}2,0 \\
(2,7)\end{array}$ & $\begin{array}{c}1,7 \\
(2,6)\end{array}$ & $\begin{array}{c}1,7 \\
(2,7)\end{array}$ \\
\hline IC $95 \%$ & $(3,3 ; 4,5)$ & $(2,9 ; 4,2)$ & $(2,3 ; 3,5)$ & $(1,7 ; 3,1)$ & $(1,5 ; 3,0)$ & $(1,0 ; 2,1)$ & $(1,2 ; 2,6)$ & $(1,3 ; 2,7)$ & $(1,0 ; 2,4)$ & $(0,9 ; 2,4)$ \\
\hline $\begin{array}{l}\text { Mediana } \\
\text { (min./máx.) }\end{array}$ & $\begin{array}{c}3,5 \\
(0,0 ; 7,0)\end{array}$ & $\begin{array}{c}3,0 \\
(0,0 ; 7,0)\end{array}$ & $\begin{array}{c}2,0 \\
(0,0 ; 7,0)\end{array}$ & $\begin{array}{c}1,0 \\
(0,0 ; 7,0)\end{array}$ & $\begin{array}{c}0,5 \\
(0,0 ; 7,0)\end{array}$ & $\begin{array}{c}0,0 \\
(0,0 ; 7,0)\end{array}$ & $\begin{array}{c}0,0 \\
(0,0 ; 7,0)\end{array}$ & $\begin{array}{c}0,0 \\
(0,0 ; 7,0)\end{array}$ & $\begin{array}{c}0,0 \\
(0,0 ; 7,0)\end{array}$ & $\begin{array}{c}0,0 \\
(0,0 ; 7,0)\end{array}$ \\
\hline P25 ; P75 & $(1,0 ; 7,0)$ & $(1,0 ; 7,0)$ & $(0,0 ; 6,0)$ & $(0,0 ; 5,0)$ & $(0,0 ; 5,0)$ & $(0,0 ; 3,0)$ & $(0,0 ; 4,0)$ & $(0,0 ; 4,0)$ & $(0,0 ; 2,0)$ & $(0,0 ; 2,0)$ \\
\hline
\end{tabular}

Tabla 2 Utilización de laxantes estimulantes por semana (pacientes que completan las 10 semanas) y número de días que ha utilizado los laxantes

\begin{tabular}{|c|c|c|c|c|c|c|c|c|c|c|c|}
\hline Semana $\rightarrow$ & 0 & 1 & 2 & 3 & 4 & 5 & 6 & 7 & 8 & 9 & 10 \\
\hline N & 51 & 51 & 51 & 51 & 51 & 51 & 51 & 51 & 51 & 51 & 51 \\
\hline \multicolumn{12}{|c|}{ Ha utilizado laxante } \\
\hline Si & $\begin{array}{c}49 \\
(96,1 \%)\end{array}$ & $\begin{array}{c}43 \\
(84,3 \%)\end{array}$ & $\begin{array}{c}41 \\
(80,4 \%)\end{array}$ & $\begin{array}{c}33 \\
(64,7 \%)\end{array}$ & $\begin{array}{c}29 \\
(56,9 \%)\end{array}$ & $\begin{array}{c}25 \\
(49,0 \%)\end{array}$ & $\begin{array}{c}22 \\
(43,1 \%)\end{array}$ & $\begin{array}{c}20 \\
(39,2 \%)\end{array}$ & $\begin{array}{c}21 \\
(41,2 \%)\end{array}$ & $\begin{array}{c}20 \\
(39,2 \%)\end{array}$ & $\begin{array}{c}21 \\
(41,2 \%)\end{array}$ \\
\hline P-valor & - & 0,0920 & 0,0279 & $<0,0001$ & $<0,0001$ & $<0,0001$ & $<0,0001$ & $<0,0001$ & $<0,0001$ & $<0,0001$ & $<0,0001$ \\
\hline \multicolumn{12}{|c|}{ Número de días que ha utilizado los laxantes } \\
\hline $\begin{array}{l}\text { Media } \\
\text { (DE) }\end{array}$ & $\begin{array}{c}4,0 \\
(2,4)\end{array}$ & $\begin{array}{c}3,5 \\
(2,6)\end{array}$ & $\begin{array}{c}2,8 \\
(2,6)\end{array}$ & $\begin{array}{c}2,5 \\
(2,6)\end{array}$ & $\begin{array}{c}2,1 \\
(2,7)\end{array}$ & $\begin{array}{c}2,1 \\
(2,8)\end{array}$ & $\begin{array}{c}1,4 \\
(2,3)\end{array}$ & $\begin{array}{c}1,7 \\
(2,7)\end{array}$ & $\begin{array}{c}1,8 \\
(2,6)\end{array}$ & $\begin{array}{c}1,6 \\
(2,5)\end{array}$ & $\begin{array}{c}1,7 \\
(2,7)\end{array}$ \\
\hline IC 95\% & $(3,3 ; 4,7)$ & $(2,7 ; 4,2)$ & $(2,1 ; 3,5)$ & $(1,7 ; 3,2)$ & $(1,3 ; 2,9)$ & $(1,3 ; 2,9)$ & $(0,8 ; 2,0)$ & $(0,9 ; 2,5)$ & $(1,0 ; 2,5)$ & $(0,9 ; 2,3)$ & $(0,9 ; 2,4)$ \\
\hline P25 ; P75 & $(2,0 ; 7,0)$ & $(1,0 ; 7,0)$ & $(1,0 ; 5,0)$ & $(0,0 ; 4,0)$ & $(0,0 ; 4,0)$ & $(0,0 ; 5,0)$ & $(0,0 ; 2,0)$ & $(0,0 ; 4,0)$ & $(0,0 ; 4,0)$ & $(0,0 ; 200)$ & $(0,0 ; 2,0)$ \\
\hline P-valor & - & 0,3001 & 0,0263 & 0,0032 & 0,0002 & 0,0002 & $<0,0001$ & $<0,0001$ & $<0,0001$ & $<0,0001$ & $<0,0001$ \\
\hline
\end{tabular}

El valor de la p se expresa versus nivel basal. 


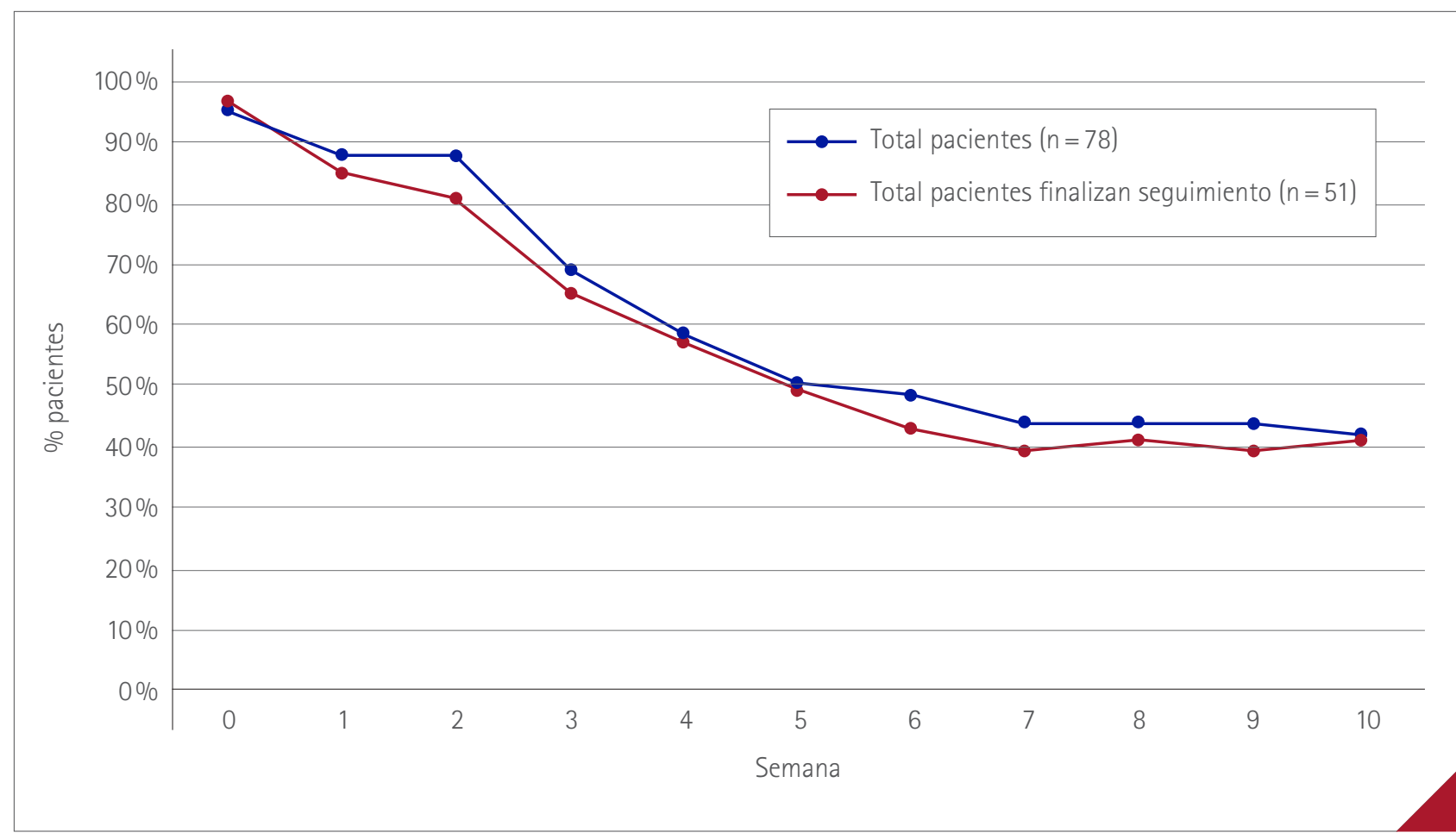

Figura 4 Utilización de laxantes estimulantes por semana (\% de pacientes)

En relación con la frecuencia defecatoria, se observó que fue bastante homogénea $(5,8[ \pm 2,2]$ nivel basal vs. $6,3[ \pm 1,9]$ semana 10$)$. Al igual que la consistencia media de las deposiciones, que no sufrieron grandes cambios: $3,7( \pm 1,1)$ semana basal a $4,1( \pm 1,5)$ en la semana 10 (figura 4).

En cuanto a los sintomas gastrointestinales se observó una disminución de los mismos en la semana 10 comparado con la basal (tabla 3).

Tabla 3 Sintomas durante la deposición durante la semana 1 del tratamiento versus semana 10

\begin{tabular}{|l|c|c|}
\hline Síntomas & $\begin{array}{c}\text { Semana 1 del } \\
\text { tratamiento } \\
(\mathrm{n}=80)\end{array}$ & $\begin{array}{c}\text { Semana 10 del } \\
\text { tratamiento } \\
(\mathrm{n}=58)\end{array}$ \\
\hline Distensión abdominal & $15(18,8 \%)$ & $10(17,2 \%)$ \\
\hline Movimientos intestinales & $36(45,0 \%)$ & $22(37,9 \%)$ \\
\hline Dolor abdominal & $32(40,0 \%)$ & $12(20,7 \%)$ \\
\hline Esfuerzo prolongado excesivo & $38(47,5 \%)$ & $15(25,9 \%)$ \\
\hline Malestar abdominal & $29(36,3 \%)$ & $8(13,8 \%)$ \\
\hline Defecación insatisfactoria & $45(56,3 \%)$ & $13(22,4 \%)$ \\
\hline Otro & $14(17,5 \%)$ & $4(6,9 \%)$ \\
\hline
\end{tabular}

\section{Aceptabilidad}

Se agrupan en diferentes categorías (nada/casi nada/ poco/algo/bastante/mucho/muchísimo) los resultados de las preguntas que se realizaron en el cuestionario a pacientes, así:

Agrupando las categorías de bastante/mucho/muchísimo dentro del concepto "facilidad de uso" ésta resultó en un $98,4 \%$. La aceptabilidad del producto de un $85,5 \%$ (se tomó bien sin rechazo) y la no aparición de molestias intestinales (distensión abdominal excesiva, plenitud, etc.) se categorizó en un $50 \%$ en estas tres categorías conjuntas.

La textura y el sabor de la fibra fue considerado como buena en un $93,5 \%$ y $80,6 \%$, respectivamente y un $61,9 \%$ indicó que recomendaría el producto.

\section{Eventos adversos}

El $60,9 \%$ de los pacientes no presentaron ningún evento adverso. Por el contrario, un 9,2\% dejó de tomar el suplemento de fibra debido a molestias relacionadas: flatulencia $(8 \%)$, dolor abdominal $(2,3 \%)$ y aerofagia $(1,1 \%)$.

Hubo un único paciente con un evento adverso grave relacionado con el producto y que finalmente dejó la medicación. El paciente reportó una hipertensión arterial no controlada con una causalidad probable con Casenfibra ${ }^{\circledR}$. En este caso la fibra pudo interferir en la absorción oral del hipertensivo debido a una interacción local a nivel intestinal. 


\section{DISCUSIÓN}

Aunque pueda parecer una contradicción, el uso indebido de ciertos laxantes puede ocasionar estreñimiento crónico. Este hecho se relaciona con mayor frecuencia en pacientes que se automedican y aquellos que presentan trastornos alimentarios.

Los laxantes estimulantes son los más utilizados y los que más abuso presentan, incluyendo tanto aquellos adquiridos mediante prescripción médica o recomendación farmacéutica como los utilizados para automedicación. Las principales razones de este abuso son la ausencia de necesidad de prescripción, el amplio conocimiento por parte de la población general debido a la publicidad y su bajo coste. Hay que añadir que se les considera inocuos y no lo son (17).

A pesar de su frecuente uso, se han realizado pocos estudios randomizados y controlados con placebo para determinar su eficacia y seguridad durante un mes de tratamiento. Estos estudios demuestran un significativo incremento de la frecuencia defecatoria y mejora de la consistencia de las heces a favor de bisacodilo y picosulfato de sodio vs. placebo. Aunque, no podemos dejar de resaltar que hubo una alta respuesta a placebo de un modo relevante (46 a un $54 \%$ ). Los eventos adversos fueron generalmente leves pero muy frecuentes, hasta un $72 \%$ (7).

Los eventos adversos y la toxicidad de los laxantes estimulantes incluyen habitualmente estreñimiento (18) y colon catártico (19-20).

Aún no hay un método recomendado para realizar una retirada de los laxantes a personas que presentan habituación y dependencia. Sin embargo, es importante que la misma preserve una función intestinal normal (21).

Las modificaciones del hábito de vida deben constituir los primeros pasos en el tratamiento del estreñimiento crónico (22). El presente trabajo evalúa la idoneidad de un suplemento de fibra fermentable y soluble complementario a la dieta, no farmacológico. Es el primer trabajo conocido hasta la fecha que evalúa este cambio de tratamiento.

En el estudio se utilizaron parámetros habituales para medir el estreñimiento del paciente en las consultas de atención primaria. Se observó desde la primera semana del tratamiento una disminución en el uso de laxantes estimulantes y del número de días en los que fueron necesarios $(\mathrm{P}<0,02)$.

Al no haberse publicado un estudio hasta la fecha similar al nuestro no podemos hacer comparativa en cuanto a eficacia. Sin embargo, en un estudio randomizado, doble ciego controlado con placebo se demostró que Orafti ${ }^{\circledR}$ (inulina), mismo componente que contiene Casenfibra ${ }^{\circledR}$, es eficaz en personas sanas con estreñimiento crónico al mejorar significativamente la función intestinal (23). Durante la suplementación con $0 \mathrm{rafti}^{\circledR}$ se observó un aumento de la frecuencia defecatoria en comparación al placebo $(4,0$ $[2,5-4,5]$ vs. $3,0[2,5-4,0]$ defecaciones/semana, $p=0,038)$.

Una revisión de los estudios que comparaban la suplementación de fibra con placebo o con otras alternativas en pacientes adultos con estreñimiento crónico y síndrome del intestino irritable (SII) mostró que la suplementación con fibra es beneficiosa en los casos de estreñimiento crónico leves y moderados (Nivel II de evidencia, Grado B) (24). Por último, en la revisión de Okawa et al. se destaca la eficacia de psyllium en el tratamiento de los síntomas del SIl y del estreñimiento funcional al aumentar el número de defecaciones completas y mejorar la puntuación en la escala de Bristol (25).

En nuestro estudio, el 94,2\% de los pacientes a nivel basal habian utilizado, al menos, un laxante durante la última semana. De los 51 pacientes que terminaron las 10 semanas de tratamiento, menos de la mitad de los pacientes (41\%) requirieron alguna dosis de laxante estimulante durante la última semana del estudio. Hubo una mejora significativa en la disminución de los laxantes utilizados al final del estudio y un $59 \%$ de los pacientes consiguieron dejar de tomarlos.

Nuestros resultados aportan información desconocida hasta el momento sobre el potencial uso de esta fibra como cotratamiento del estreñimiento en pacientes que usan crónicamente laxantes estimulantes.

En este estudio se seleccionó una fibra basada en una mezcla de fibras solubles (fructooligosacáridos y maltodextrina resistente a la digestión), pero con distinto grado de fermentabilidad. La fibra seleccionada presenta ventajas organolépticas. No crea una disolución viscosa, es soluble en agua, es incolora, inodora e insípida, con lo cual es muy fácil de tomar y así se ha visto reflejado en los resultados de aceptabilidad.

La ventaja de esta fibra radica en que no crea habituación, puede ser ingerida por todo tipo de pacientes y su efecto adverso más frecuente son las molestias gastrointestinales que suelen ir remitiendo (26). Además, la fibra presenta un efecto prebiótico gracias a su fermentación colónica (22).

La fortaleza más importante del estudio es la novedad que presenta en relación con su ámbito de realización: la farmacia comunitaria con un seguimiento prospectivo. Las debilidades son las comunes a cualquier tipo de estudio observacional: al no haber grupo control, no se puede sustraer el posible efecto placebo, aunque es cierto que cada sujeto ha sido control de sí mismo, lo que nos permite comparar datos. 


\section{CONCLUSIÓN}

La alta frecuencia de estreñimiento hace pensar que esta fibra podría ser una alternativa válida para aquellos pacientes que están con tratamiento crónico con laxantes estimulantes, con el fin de eliminar la dependencia y el estreñimiento asociado debido al uso inadecuado de los mismos.

\section{AGRADECIMIENTOS}

Los autores quieren agradecer a todas las farmacias que han formado parte de este estudio por su implicación en el mismo debido a la dificultad a la hora de reclutar los pacientes: Farmacia Maica Lluch Villamor, Farmacia Terricabras CB, Farmacia Neus Girón Monge, Farmacia Mercedes Ferro Mauri, Farmacia Anna María Pi Culell, Farmacia Teresa Roca de Haro, Farmacia Dolors Grima Aulet, Farmacia Elda Llobet Alonso, Farmacia Beatriz Izquierdo, Farmacia Álvaro Gil Tarragato, Farmacia Ángeles Nebot Rico, Farmacia Susana Bereciartúa, Farmacia Jesús Serrano Matoses, Farmacia Rosa María Rubio Gómez, Farmacia Susana Camacho Díaz, Farmacia Monte del Pilar, Farmacia Valdelasfuentes.

\section{REFERENCIAS BIBLIOGRÁFICAS}

1. Douglas A. Drossman, editor. Rome IV Diagnostic Algorithms for Common GI Symptoms. 2nd ed. Rome Foundation, Inc.: 2017.

2. Rey E, Balboa A, Mearin F. Chronic constipation, irritable bowel syndrome with constipation and constipation with pain/discomfort: Similarities and differences. Am J Gastroenterol. 2014;109(6):87684. doi:10.1038/ajg.2014.18

3. Wong RK, Palsson OS, Turner MJ, et al. Inability of the Rome III criteria to distinguish functional constipation from constipation-subtype irritable bowel syndrome. Am J Gastroenterol. 2010;105(10):222834. doi:10.1038/ajg.2010.200

4. Bharucha $A E$, Locke $G R$, Zinsmeister AR, Seide BM, McKeon $K_{1}$ Schleck CD, Melton $\sqcup$ III. Differences between painless and painful constipation among community women. Am J Gastroenterol. 2006 Mar;101(3):604-12. Disponible en: dhttps://journals.Iww.com/ajg/ Abstract/2006/03000/Differences_Between_Painless_and_Painful.30.aspx

5. Kamm MA, Muller-Lissner S, Talley NJ, et al. Tegaserod for the treatment of chronic constipation: A randomized, double-blind, placebo-controlled multinational study. Am J Gastroenterol. 2005;100(2):362-72. Disponible en: https://journals.Iww.com/ajg/ Abstract/2005/02000/Tegaserod_for_the_Treatment_of_Chronic.17.aspx

6. Garrigues V, Galvez C, Ortiz V, et al. Prevalence of constipation: agreement among several criteria and evaluation of the diagnostic accuracy of qualifying symptoms and self-reported definition in a population-based survey in Spain. Am J Epidemiol. 2004;159:520526. doi:10.1093/aje/kwh072

7. Noergaard M, Traerup Andersen J, Jimenez-Solem E, Bring Christensen M. Long term treatment with stimulant laxatives - clinical evidence for effectiveness and safety. Scand J Gastroenterol. 2019;54(1):27-34. doi:10.1080/00365521.2018.1563806

8. Anónimo. Final report on the safety assessment of Ricinus Communis (Castor) Seed Oil, Hydrogenated Castor Oil, Glyceryl Ricinoleate,
Glyceryl Ricinoleate SE, Ricinoleic Acid, Potassium Ricinoleate, Sodium Ricinoleate, Zinc Ricinoleate, Cetyl Ricinoleate, Ethyl Ricinoleate, Glycol Ricinoleate, Isopropyl Ricinoleate, Methyl Ricinoleate, and Octyldodecyl Ricinoleate. Int J Toxicol. 2007;26 Suppl 3:31-77. doi:10.1080/10915810701663150

9. Harris M. Scott. Evaluation and treatment of constipation. International Foundation for Functional Gastrointestinal Disorders. 2004. Disponible en: https://iffgd.org/wp-content/uploads/118_Constipation_UPDATED.pdf

10. Agencia Española de Medicamentos y Productos Sanitarios. Ficha técnica Dulcolaxo Bisacodilo. Disponible en: https://cima.aemps.es/ cima/pdfs/es/ft/28410/FT_28410.pdf

11. Agencia Española de Medicamentos y Productos Sanitarios. Ficha técnica Aceite de ricino ORRAVAN. Disponible en: https://cima. aemps.es/cima/pdfs/es/ft/34912/FT_34912.pdf

12. Agencia Española de Medicamentos y Productos Sanitarios. Ficha técnica Cáscara sagrada ARKOPHARMA. Disponible en: https://cima. aemps.es/cima/dochtml/ft/74097/F__74097.html

13. Agencia Española de Medicamentos y Productos Sanitarios. Ficha técnica Sen ARKOPHARMA. Disponible en: https://cima.aemps.es/ cima/pdfs/es/ft/72278/FT_72278.pdf

14. Agencia Española de Medicamentos y Productos Sanitarios. Ficha técnica Gelax Plantago/Frángula Polvo Oral. Disponible en: https:// cima.aemps.es/cima/pdfs/es/ft/77955/FT_77955.pdf

15. Rutter K, Maxwell D. Diseases of the alimentary system. Constipation and laxative abuse. Br Med J. 1976;2(6042):997-1000. doi:10.1136/ bmj.2.6042.997

16. Roerig JL, Steffen KJ, Mitchell JE, Zunker C. Laxative abuse: epidemiology, diagnosis and management. Drugs. 2010;70(12):1487-503. doi:10.2165/11898640-000000000-00000

17. Hallmann F. Toxicity of commonly used laxatives. Med Sci Monit. 2000;6(3):618-628. Disponible en: https://www.medscimonit.com/ abstract/index/idArt/421298

18. Cooke WT. Laxative abuse. Clin Gastroenterol. 1977;6:659-73. doi: 10.1016/S0300-5089(21)00182-6

19. Baker EH, Sandle Gl. Complications of laxative abuse. Annu Rev Med. 1996; 47:127-34. doi:10.1146/annurev.med.47.1.127

20. Kim SK, Gerle RD, Rozanski R. Cathartic colitis. AJR Am J Roentgenol. 1978;131:1079-81. doi:10.2214/ajr.131.6.1079

21. Schuster BG, Kosar L, Kamrul R. Constipation in older adults: stepwise approach to keep things moving. Can Fam Physician. 2015 Feb;61(2):152-8. PMID: 25676646; PMCID: PMC4325863. Disponible en: https://www.cfp.ca/content/61/2/152.long

22. Simpson HL, Campbell BJ. Review article: dietary fibre-microbiota interactions. Aliment Pharmacol Ther. 2015;42(2):158-179. doi:10.1111/apt.13248

23. Micka A, Siepelmeyer A, Holz A, Theis S, Schön C. Effect of consumption of chicory inulin on bowel function in healthy subjects with constipation: a randomized, double-blind, placebo-controlled trial. Int J Food Sci Nutr. 2017;68(1):82-89. doi:10.1080/09637486.2016.1 212819

24. Rao SS, Yu S, Fedewa A. Systematic review: dietary fibre and FODMAP-restricted diet in the management of constipation and irritable bowel syndrome [published correction appears in Aliment Pharmacol Ther. 2015 Aug;42(4):490]. Aliment Pharmacol Ther. 2015;41(12):1256-1270. doi:10.1111/apt.13167

25. Okawa Y, Fukudo S, Sanada H. Specific foods can reduce symptoms of irritable bowel syndrome and functional constipation: a review. Biopsychosoc Med. 2019;13:10. Published 2019 May 8. doi:10.1186/ s13030-019-0152-5

26. Portalatin M, Winstead N. Medical management of constipation. Clin Colon Rectal Surg. 2012;25(1):12-19. Disponible en: https://www.ncbi. nlm.nih.gov/pmc/articles/PMC3348737/pdf/ccrs25012.pdf 